\section{A (Uliniral Study}

\author{
OF
}

\section{THREE CASES OF HEART-BLOCK.}

BY

T. WARDROP GRIFFITH, C.M.G., M.D., F.R.C.P., PROFESSOR OF MEDICINE, UNIVERSITY OF LEEDS ; HONORARY PHYSICIAN, GENERAL INFIRMARY AT LEEDS.

THE three cases of heart-block which form the subject of this communication present certain features of interest. In the first, a block which remained complete for a long period suddenly broke into a two to one block and ultimately passed off altogether. In the second case, though the vexed and difficult question of whether the block was partial or complete arose from time to time, there certaidly were periods when the block was of the former cliaracter and it was mainly during these that the tracings I discuss here were taken. The phenomenon of ventricular "escape" is shown; an auscultatory sign is mentioned to which $I$ have elsewhere referred 1 the effects of amyl nitrite and of atropine are discussed, and tracings are shown which demonstrate the occasional occurrence of "flutter" of the auricle. The third case is put on record not only as an example of complete heart-block with an unusually slow ventricular rhythm, but on account of the large number of attacks of the Adams-Stokes character, during some of which satisfactory tracings were obtained. In this case also, simultaneous tracings of the pulse and of the respiration showed a remarkable relation between the periods of asystole and those of the apnoeic phases of the CheyneStokes respiration when that was present.

\section{Case I.}

W. R., aged 60, a gardener, was admitted under the care of my colleague, Dr. Watson, to whom I was indebted for permission to study the case and who has also kindly allowed me to publish the results of my investigations. Four months before admission he felt giddy when at work; this feeling was transient, but recurred in a fortnight and then almost every day. He became very conscious of the beating of his heart and volunteered the information that its action lad become very infrequent. He was a temperate man and moderate in the use of tobacco. He had had rheumatic fever when he was 38 years of age; there was no evidence of syphilis. His complaint was of some measure of breathlessness and of a feeling of oppression over the heart. The arterial walls were thickened; the apex beat was forcible and it was external to the nipple line a systolic bruit was audible with its maximum at the apex and wasalso heard at the aortic and pulmonary areas, but there was no clear evidence of a valvular lesion. The pulse was regular and infrequent from the date of his admission till the twentyeighth day of his stay in hospitol the palse never exceeded 36 . and it was of histed as low and it was counted as low as 24. On the twenty-eighth day of his stay in hospital he detected a change in the frequency of the heart's action. On counting the pulse he found it was 48 ; shortly before he had counted it at 24 , and his report at a later period of the same day was that " it was running backwards and forwards from 44 to 68 and 70 and upwards, but was never keeping to one regular beat."

The tracings shown in Figs. 1 to 5 were talen on the same day during the former of these two phases. The
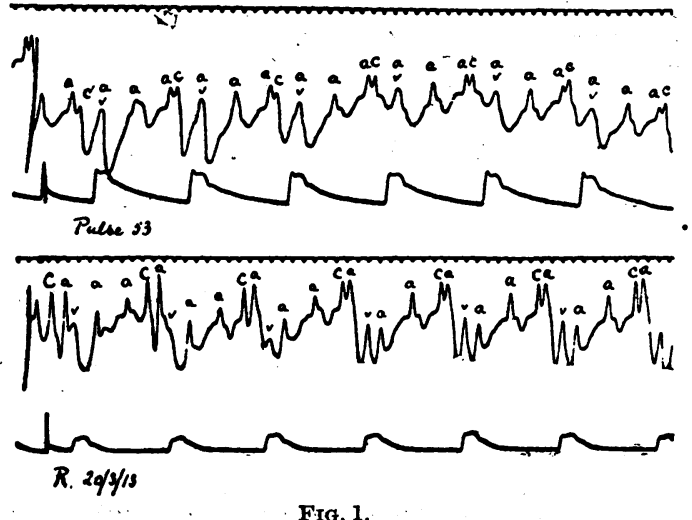

Fra. 1.

upper tracing of Fig. 1 might readily be interpreted as an example of a partial block in which every third auricular stimulus passes to the ventricle. The lower tracing, taken by itself, might also bear that interpreta. tion, though an undoubted lengthening out of the $a-c$ interval as the tracing is read from left to right ought to arouse one's suspicions of the block being a complete one. When, however, the upper and lower tracings are viewed in relation to one another, the evidence in favour of the block being a complete one is very strong. The $a-c$ interval is much longer in the lower than in the upper tracing, and a partial block would not account for this. On the other hand, if the block were a complete one it is clear that a temporary acceleration of the auricular rate would result in this interval being lengthened. The diagram in Fig. 2 explains this.

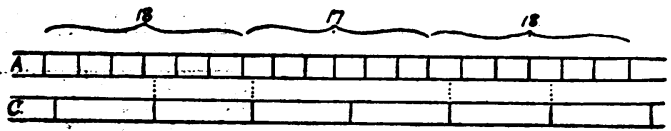

FIG. 2.

I have elsewhere ${ }^{1}$ discussed the difficulties, sometimes I believe insuperable, which await the attempt to dis. tinguish between a partial and a complete auriculo. ventricular block. In. this case it is not a difficult task. If, in any case where the question arises of there being a two to one or a three to one heart-block, a long tracing is taken, and it is found that variations in the frequency of the auricular contractions are slavishly followed by similar variations in the ventricular rate, this will be accepted as confirming the partial nature of the block. If, on the other hand, in spite of variations in the auricular rate, the ventricle goes on the even tenor of its way and beats with perfect regularity, we may regard the block as being completê. It is true that in cases of complete block there is nothing to prevent the auricular rate happening to be exactly twice or three times that of the ventricular rate, but the arm of coincidence would require to be a very long one for this relationship not to vary in a tracing taken over a period of many minutes. Still I have seen it occur, and it is sometimes a good plan to get the patient gently to inhale some ammonia, which lias the effect of increasing the auricular rate. Light may thus be thrown on the relationship between the auricular and ventricular contractions. Fig. 3 shows the result of this
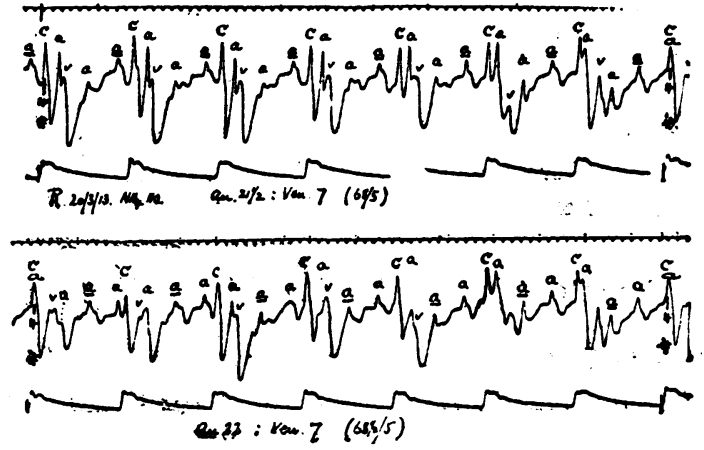

FIG. 3.

experiment. At a time when the auricle was beating exactly three times the rate of the ventricle, the patient was directed to inhale a little ammonia. For some time, but only for the period of a few beats, the auricle went on beating at the same rate; then the beats became rather more frequent. As it was observed that the ventricular rate was not affected in the slightest degree, confirmation was obtained of the complete character of the block. In the upper tracing of Fig. 3 it will be seen that every third auricular wave-and this I have under. lined-" falls back" from the "c" which comes after it, and in this way the seven radial periods between the two asterisks are equal to twenty-one and a half interauricular periods. With the increase in auricular rate in the lower tracing it is seen that seven radial periods correspand to twenty-two interauricular periods. Throughout, the ventricle beats with remarkable regularity.

But in this case the completeness of the block was obvious without artificial means. Later in the day the 
tracing shown in Fig. 4 was obtained. The block is manifestly a complete one, and it will be seen that at every fourth cycle auricle and ventricle contract at the

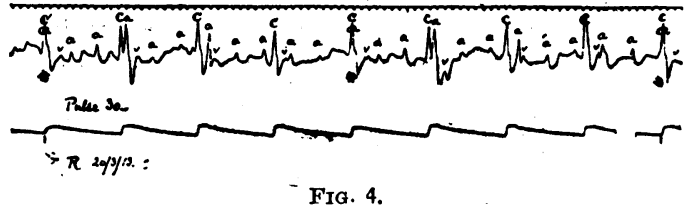

same moment. While this relationship prevailed a tracing was taken over the apex beat, and this is shown in Fig. 5. The position of some of the auricular waves is open to doubt, but in the case of the second and sixth ventricular

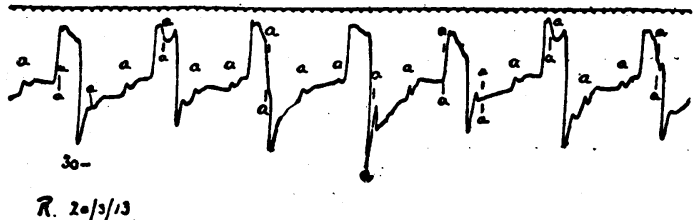

Fig. 5.

impulses it is clear that the systolic plateau is dimpled by the drag of the contracting auricle, while in the case of the third and seventh the end of the plateau is modified. Some may sce a difficulty in accepting the view that the plateau of a powerfully contracting ventricle can be dimpled by the action of the auricle, but this dimpling of the plateau does not represent any victory of the auricle over the ventricle. Here there is no question of which is the stronger; the greater part of the ventricular force is engaged in driving blood into the aorta, a small part of it only in maintaining the forward thrust of tho apex. This contracting ventricle is drawn back as a whole from the chest wall by the drag of the auricle in systole.

The sudden increase in the pulse rate noticed by the patient on the twenty-eighth day of his stay in hospital has been mentioncd; for about thirty-six hours the rate varied from about 36 to 68 or 70 . On the day on which the alteration in rate was obscrved the tracing shown in Fig. 6 was obtained. Calculated from the slow rhythm

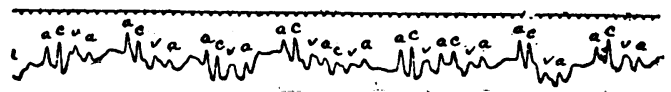

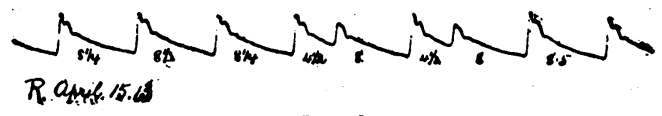

Fig. 6.

the pulse rate is seen to be about 36 per minute. If the condition is still one of completo block, we must regard the fifth pulse wave as the expression of an extra-systole of the kind described by Wenckebach in complete heartblock; and this view would be strengthened by there being no lengthening of the $a-c$ interval which leads to this fifth systole as might be expected if two auricular stimuli got through in succession, as well as by the next radial period being practically equal to the customary long radial intervals. Still $I$ incline to thinl that the condition is really one of a two to one heart-block with the occasional passage of two auricular stimuli in succession, and this

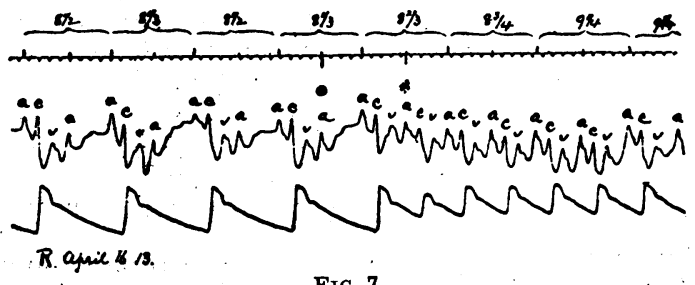

Fig. 7.

appears to be borne out by the two tracings which I took the following day. Figure 7 shows what I take to be a change from a two to one rhythm to one in which every anricular stimulus passes to the ventricle. Calculated on tho slow rate the pulse is about 36 ; when every stimulus gets through the auricular rate very soon falls slightly, and this is reflected in the rate of the ventricle, for towards the end of the tracing it will be seen that the time

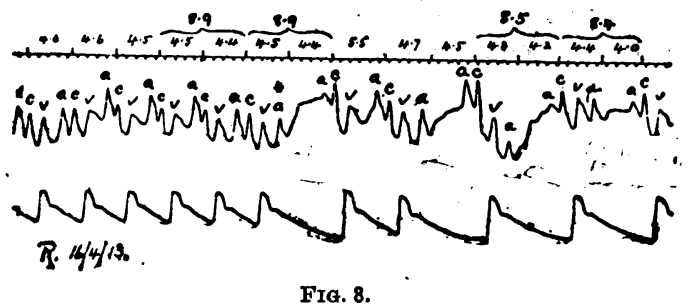

occupied by two radial periods is rather greater than that occupied by one period of the slow rate. Figure 8 shows the converse of this-a change, that is to say, from the fast rhythm to the slow. Here it is to be observed that shortly after the blocking of every second stimulus from the auricle, the auricular rate increases. I think that in these variations we may see an expression, though at firstsight not perhaps a very obvious one, of the well known relationship between the blood pressure in the medulla and the frequency of the cardiac action. When the blood pressure rises the cardiac systoles are lessened in number by vagus influence; when it falls, the removal of that influence leads to their increase. This influence of the vagus is of course exercised primarily on the auricle, and it is the rhythm of this chamber which must be studied in this connexion. In Fig. 7 the sudden increase in the number of systoles due to the passing of the block will tend to raise the blood pressure; this in turn will lead to a slowing of the auricle, which will result in two radial pulse periods of the rapid phase being slightly greater than one of the slow phase. So also in Fig. 8 the blocking of auricular stimuli after the middle of the tracing will primarily tend to lower arterial blood pressure, and the quickening up of the auricle which soon follows may be regarded as being the result of this, and also an adaptive effort to combat the lowering of pressure.

Two days afterwards the condition was that shown in Fig. 9. Every auricular stimulus passes through, but there remains some lengthening of the $a-c$ interval. There was no recurrence, and the man went home after a few days of this normal rhythm. I heard from his
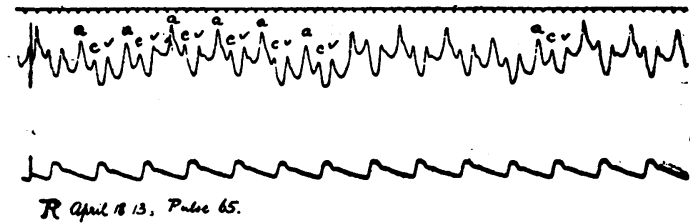

FiG. 9.

medical man some time afterwards that he had again had an infrequent pulse for some three weeks; that this suddenly passed off, the pulse rising to 120 and then in ten minutes falling to 80 . He died some months after this without any obvious cardiac symptoms, and I understand that he had abdominal discomfort and emaciation.

\section{Case II.}

A woman of about 38, who was admitted under my care complaining of dyspnoea and who was conscious of the cardiac action. The systolic blood pressure was $250 \mathrm{~mm}$. of mercury and sometimes higher, and there was a trace of albumin in the urine. The pulse was infrequent, sometimes regular and sometimes irregular. The degree of irregularity was somesometimes ir to strite one at once; and on some occasions it was so slight that it could not be detected without instrumental was so

The first tracing which I show from this patient (Fig. 10) reveals an impairment of conductivity, for not only is every second auricular stimulus bloclred, but the passago of those which reach the ventricle is delayed. I find it is often an advantage to measure from the summit of the " $a$ " wave to the radial rise in estimating conductivity rather than to adopt the usual measurement from tho beginning of the auricular wave to the beginning of the carotid. ${ }^{1}$ For reasons which I need not mention here tho beginnings of both these waves may be "obscured: It is clear that the block is not a complete one and that tho auricular rate is dominating the ventricle. In the ersit 
place the ventricular rate is 47 , which is high for a complete block, and in the second place the relationship which is shown in the figure between auricle and rentricle was preserved over a long tracing.

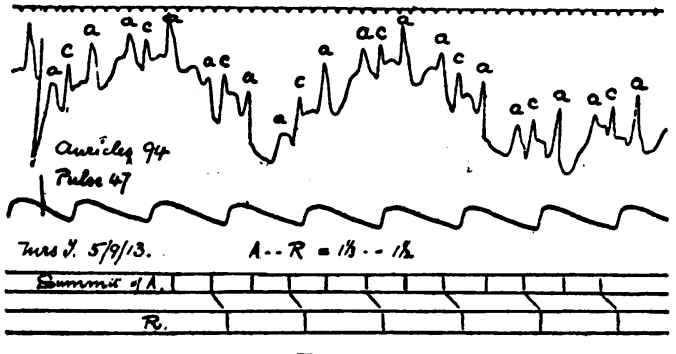

FIG. 10.

In the next tracing (Fig. 11) the auricle is beating less frequently-that is to say, at 54 and not at 94 a minuie. At the time this tracing was made most of the auricular stimuli got through in spite of the fact that some of them were premature, a condition inimical to transmission ; but

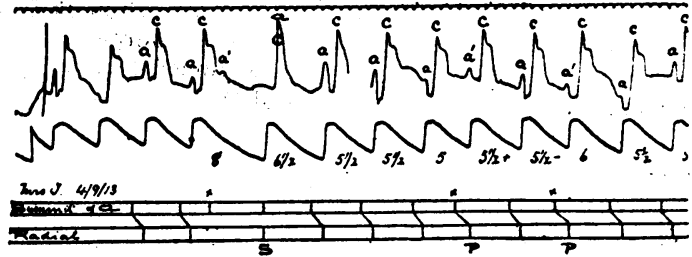

Fig. 11.

at one point it will be seen that a contraction of the auricle has indeed failed to reach the ventricle, probably because it is more premature than the others which are only asso. ciated with an increase in the $a-c$ interval. With the auricle beating at 54 instead of at 94 the blocking of one stimulus from the auricle will give the ventricle a much longer time in which to build up its stimulus material to the flash point, and this is more than enough, for its systole occurs eight fifths of a second after the previous beat-that is, as it so happens, at the same time as the auricle contracts, and of course at a time that it cannot be attributed to this event. This plenomenon is not un. common in cases of partial block and is by Einthoven referied to as "ventricular escape."

The next tracing (Fig. 12) shows the blocking of stimuli becoming rhythmical. One of these is premature. The

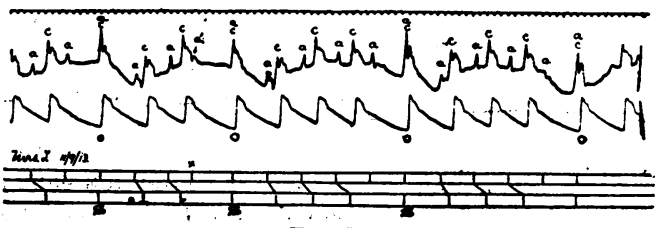

FIG. 12.

escaped ventricular contraction occurs synchronously with the auricular contraction, and this gave rise to an altera. tion in the first sound of the heart. There was usually a faint systolic bruit at the apex, but when in consequence of ventricular escape auricle and ventricle contracted at the same time the first sound became lond and thumping and the bruit was not audible. When in a case of infre. quent cardiac action the first sound shows this alteration, either rhythmically or irregularly, one may strongly suspect either a partial block with escape of the ventricle or a condition of complete block. The black dots under the radial tracing indicate beats which were announced as being loud by an observer who was confining his attention to that one point: He did not give his signal, however, at one point when the two cavities contracted together, and this I have indicated by a small circle.

\section{i. Effect of Amyl Nitrite.}

As I had found that inhalation of amyl nitrite caused a partial block to be overcome, I decided to make the test in this case, but on examining the patient betore doing so I found the heart so nearly regular that I was unable to make out the slight irregularity which existed without instrumental means. I also found that the characters of the pulse in the veins of the neck had entirely changed (see Fig. 13). The waves referable to the auricle were now 266 per minute, while the ventricle was beating so

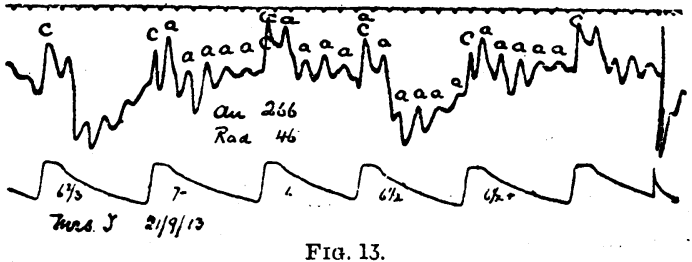

infrequently that the pulse was about 46 and the radial spaces varied from about six to seven fifths of a second The condition was clearly that known as "flutter," and for reasons I need not mention at present I am in some doubt whether the block was a complete or a partial one. Inhalation of amyl nitrite did not at first produce any effect on the auricle, which continued to give rise to 266 waves per minute. The raclial pulse increased to 55 (see Fig. 14). If the block was a partial one this might
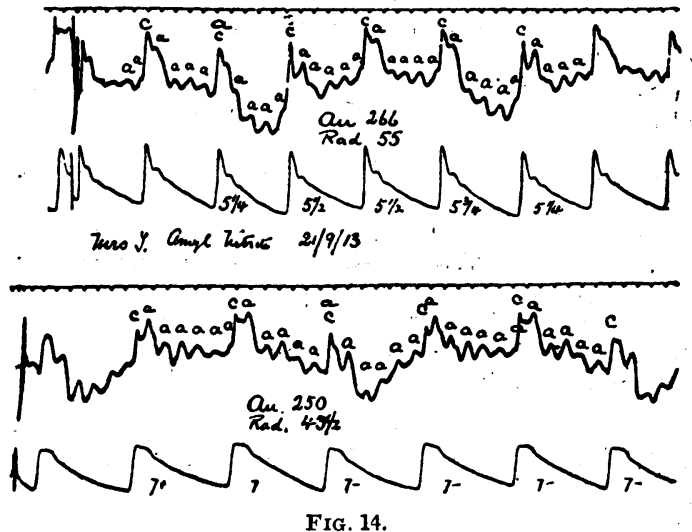

be due to auricular impulses getting through more fre. quently from the improvement in conductivity which occurs from amyl nitrite; if, on the other hand, the block was a complete one, the action on the ventricle must be of a more direct nature. In a few seconds the auricular waves fell to 250 and the pulse to $43 \frac{1}{2}$ per minute.

I waited till this condition of auricular flutter had passed off before I considered that I had an opportunity of trying
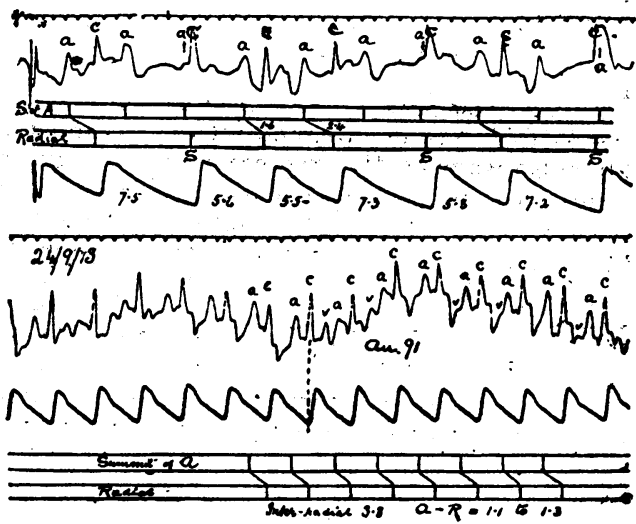

FIG. 15.

the effect of ampl nitrite on conductivity. In the upper tracing of Fig. 15 it will be seen that the auricle had resumed its normal action; it was beating at 65 ; there were escaped beats of the ventricle following on blocking of stimuli, and the $a-c$ interval varied from 1.6 to 2.4 fifths of a second. Inhalation of amyl nitrite at once increased the auricular rate to 91 per minute. Yet in spite of this great demand on the conducting function of the bundle every impulse was transmitted; not only so, but the time of transmission was lessened, as will be seen on comparing the two tracings. The next tracing (Fig. 16) shows thd the effect on the conducting function of the bundle began to pass off before the effect on the auiricular rate; tor 
while this remains at 91 we find a progressive lengthening of the $a-c$ interval until this culminates in a block. This tracing a!so gives evidence that the inhalation of amyl

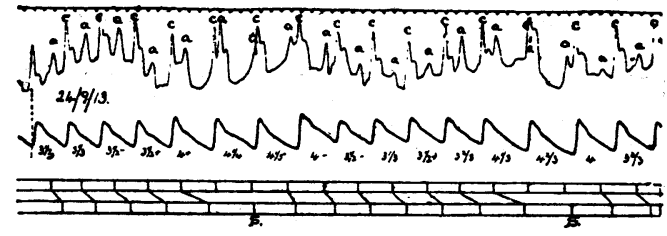

FIG. 16.

nitrite increases the rate of the idio-ventricular rhythm, for it appears that if the ventricle is left to itself it enters into contraction at the end of a period of about four and a half fifths of a second.

\section{Effect of Atropine.}

On another day I tried the effect of atropine on the function of conductivity. However, on taking a preliminary tracing $I$ found that the auricle was again in a condition of flutter. This passed off in about an hour, and the two tracings seen in Fig. 17 were taken. The
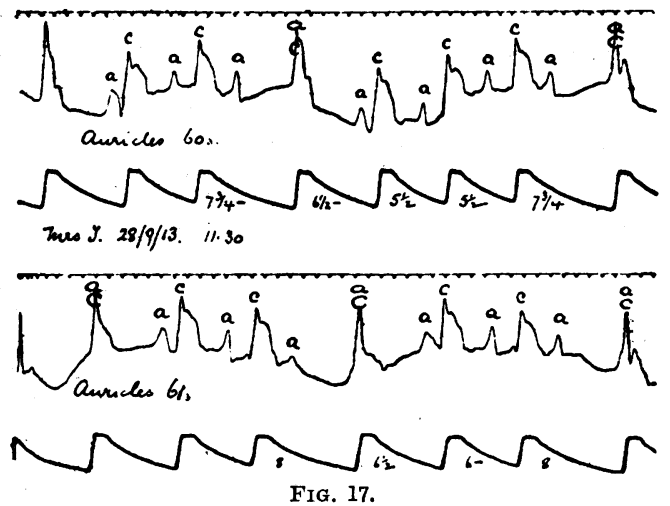

auricles were beating at 60 and 61 , and every fourth or fifth stimulus was blocked. One-fiftieth of a grain of atropine sulphate was then injected subcutaneously; in fifteen minutes all the stimuli from the auricle passed through to the ventricle. This is seen in the upper tracing of Fig. 18. Some impairment of conductiviiy is indeed

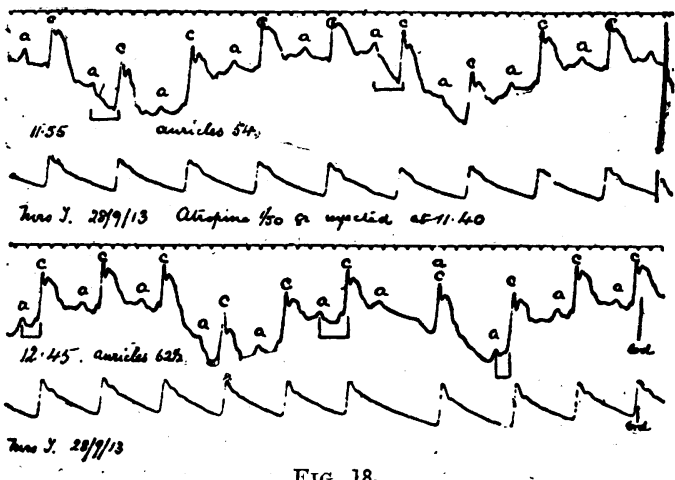

still apparent in the lengthened $a-c$ interval, which slightly exceeds the lcngest intervals in the previous tracing, but in view of the fact that every stimulus is now passing the comparison is not quite a fair one. It will be noted that the auricles are now beating at 54 instead of at 60 . This is not the first time I have found atropine produce this effect in the earlier stage of its action, ${ }^{2}$ and it might be suggested that the passing off of the block is attributable to a lessened demand on the function of the conducting structures in consequence of this slowing of rhythm. The lower tracing gives the answer to this, because here the auricular rate has gone up to rather more than it was before the injection, and yet, though there is some return of blocking of stimuli, there are long runs where every stimulus reaches the ventricle. Here also, as with amyl nitrite, the effect of the drug is more evanescent in respect of conductivity than in respect of the auricular rate.
Sugsequent History of Case ir.

The patient remained in hospital many montlis, and a great many tracings were taken. A very comman rhythm was that shown in Fig. 10, with every stimulus blocked. At other times the block was clearly a complete one, with a regular ventricular rate of 37 to 40 a rate which is in harmony with the time at which the sporadic escapes of the ventricle have been seen to occur.

About twelve months after her discharge from hospital she was again admitted, having had a sudden attack of left hemiplegia without any loss of consciousness. Both arm and leg were affected, but the weakness was not great; at first there was neither hemianaesthesia nor hemianopsia, but the hemiplegia increased and soon became associated with both these symptoms. In the course of a few days she suddenly became unconscious; very soon Cheyne-Stokes respiration came on, followed by death. During the last days of her life the tracings slioved a condition of partial block in which the passing of a stimulus was regularly followed bythe blocking of the next, which in turn led to an escape of the ventricle. In this way the radial tracing presented a series of long and short periods, the long ones being rather less than two interauricular spaces, and the short ones being rather more than one interauricular space. Sliortly before she died the pulse went up to 85 , and though no venous tracing was taken there can be no doubt that, so far as the actual passing of stimuli is concerned, the block had disappeared.

CASE III.

T. M., a labourer, aged 50, was admitted under Dr. Churton, to whom I am indebted for permission to nse the case. Alcoholic excess for years; no history of syphilis. Three weeks before admission, at a time when he was not exerting himself at all, he had an attack of loss of consciousness and is said to have been convulsed. He was able to get home by himself. On admission he was found to be the subject of general atheroma; there was great enlargement of the heart; the blood pressure rose during the systole to 210 and the pulse was anacrotic. The radial pulse was regular and about 22 per minute. At the apex a systolic bruit was heard which varied very much in distinctness from beat to beat, and which at times was inaudible, the first sound being then loud and thumping. This last sign, pointing as it does to a simultaneous systole of the auricle and ventricle with som
complete heart-block.

Figure 19 shows tracings of the jugular pulse in the upper and of the apex beat in the lower record, the radial pulse
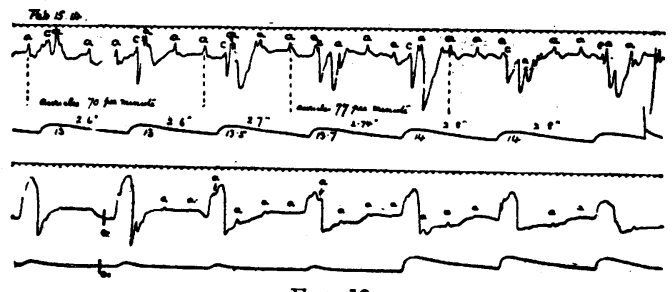

FIG. 19.

being also shown in each case. It will be seen that though the auricular rate varies from 70 to 77 , the ventricular rate is not affected; the block is a complete one. He then began to have long periods of asystole with, after some little time, tonic and clonic convulsions following on loss of consciousness. Of these attacks I saw an immense number; the majority of them conformed to the following description, which I quote from my notes made at the time:

"While lying quietly in his usurl condition, the pulse stops quite suddenly; he becomes pale, and looks as if he were going to die. I think the respiration becomes less full. Then, after a varying period, usually about fifteen seconds, he becomes unconscious. To test this I was in the habit repeat uumbers or letters after me; he would become confused in his utterance over one or two letters or numbers confused in his utterance over one or two letters or numbers, and then all response would cease. Following very soon on the loss of begins to have slight tonic spasm, the face bing say, he turned from the left towards the right and the head asualy raised from the pillow. Some alteration of expression of the face suggestive of pain is usually noticed. Then, quite suddenly. and before the disappearance of the tonic spasm, the puls, and before the disappearance of the tonic spasm, the pulso returns, the first beat as a rule being quite strong and fuld;
at once the face assumes its normal colour, and in a second or two becomes redder than usual. About the same time the tonio spasm, which lasts only a few seconds, passes off, and the hedid may again rest on the pillow. Then, almost at once, clonio 
spasms of moderate violence come on, and after these have lasted a few seconds he usually puts his bands to his head and complains in a confuset voice of pain in the head before all the spasm has passed off."

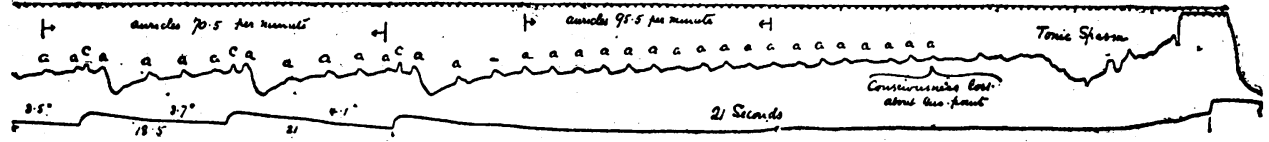

FIG. 20.

The tracing shown in Fig. 20 was taken during one of these attacks. The increase in the auricular rate during

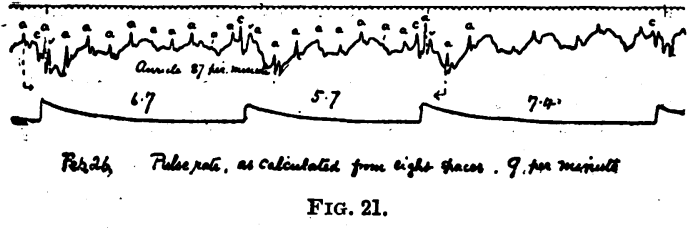

the period of asystole is well seen, and is doubtless the expression of the fall in arterial pressure. The duration of mission, tended to become less frequent, ard Fig. 21 was taken at a time when the pulse rate had fallen to 9 per minute. Sometimes long pauses of ten or eleven seconds

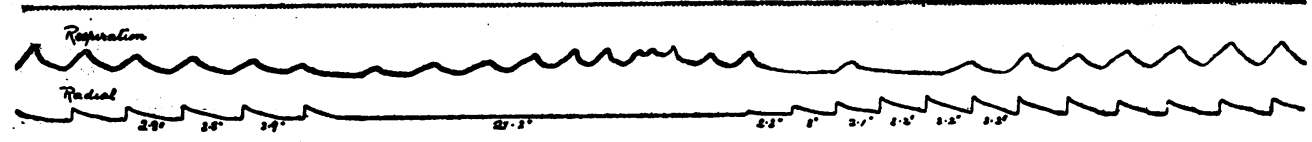

occurred, and towards the end of these pallor, without unconsciousness, prevailed.

Apparently in connexion with the long periods of asystole-and here I refer to those which were not associated with convulsion or with loss of consciousnessCheyne-Stokes respiration was present. The relation was constant and peculiar, and it is shown in Fig. 22; the period of apnoea does not begin until after that of asystole has passed off; there is sometimes an interval of a few seconds between the two phenomena; they never coincide in time. I am not aware that this relationship has been formerly noted. In this tracing the return of the pulse is of the staircase variety. I liave looked through my many

Feb 23.14

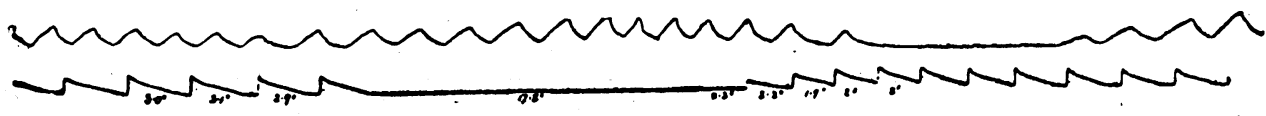

FIGriz2.

asystole is twenty-one seconds; the point of time at which consciousness was completely lost is indicated, and it will be seen that the tonic spasm interfered with the record from the venous pulse.

His pulse, which as I have said was about 22 on ad. tracings, but find, as I have already mentioned, that as a rule the pulse returns with a full beat.

1 Heart, vol. iii, No, 2,2 REFERENCES. No. 10 .

\section{A Perture}

$$
\text { ON }
$$

\section{CAPILLARY PRESSURE AND OEDEMA** BY \\ LEONARD HILL, M.B., F.R.S.}

IT is generally held, both in physiological and pathological teaching, that the transudation of fluid through the wall of the capillaries is due to the pressure of the blood within these vessels-that is to say, to the force of the heart beat. The average capillary pressure is taken as something between 20 and 30 millimetres of mercury, and it is suggested that this pressure is sufficient to overcome the osmotic force which is found to be exerted through gelatine membranes by the proteins of the blood and separate the water and crystalline substances which pass out from the capillaries into the tissues. According to the modern theory of the secretion of urine, it is assumed that such filtration takes place through the Malpighian capsules, and it is supposed that to maintain this filtration and to overcome the osmotic pressure of the colloids the pressure. within the glomerular capillaries is about 20 per cent. less than that in the aorta. The assumption that there is any such high pressure within the capillaries I believe to be erroneous.

My attention was first drawn to capillary pressure some twenty-five jears ago when $I$ was inquiring into the principles of the cerebral circulation. I measured the pressure of the brain against the skull wall by means of a tube filled with water and closed by a thin rubber membrane, which was so screwed into a trephine hole that the rubber membrane came into contact with the bulging brain; the tube was connected with a glass tube

* Given at 8t. Mary's' Hospital in the Institute of Pathology and Research during the course on Pathological Research in its Relation
to Medicine. containing an air-bubble index, and this by a T-piece with a pressure bottle and a manometer. When the tabs was screwed in and the rubber membrane came in contact with the brain, the air-bubble index was pushed out from the zero pressure position. On raising the pressure bottle, so as to restore the air bubble to the zero position, the manometer recorded the pressure required to push in the bulging brain, and restore it to its natural position within the closed skull cavity. The pressure of the brain against the skull wall is due to the distension of the cerebral arterioles and capillaries by the heart, and my method, I believe, gave me a measure of the pressure in these vessels.

In the anaesthetized animal lying in the horizontal position this pressure was about $5-10 \mathrm{~mm}$. $\mathrm{Hg}$, and was the same as the pressure measured in the torcular Herophili; in the dog this was a bony carity, and therefore suitable for the insertion of a tube and the measurement of the cerebral venous pressure. Treplining the lamina of the atlas and screwing in a tube there, I obtained the pressure of the cerebro-spinal fluid. I found the pressure of the cerebro-spinal fluid, cerebral venous pressure, and the pressure of the brain against the sliull wall were the same and varied together, and I concluded that the cerebral capillary pressure is only some $5-10 \mathrm{~mm}$. $\mathrm{Hg}$, and is practically the same as the pressure in the cerebral veins ; only the least difference is required to maintain the flow. I may point out here that whether blood is extrava. sated within the skull or we are dealing with a depressed fracture, either the blood clot or the depressed bone expresses blood out of the brain contiguous to it, and occupies the room of this blood, and so raises the circulatory pressure up to arterial pressure in adjoining parts. The clot or the bone do not continue to press, but occupy room. The increased brain pressure is due to the shutting up of the capillary-sized vessels.

The brain is enclosed within a rigid case-the slrulland the total content cannot vary, but there may be more blood in the brain and less tissue substance and cerrebro. spinal fluid on the one hand, while on the other hand 